\title{
Emerging Roles of Micrornas in Diabetic Cardiomyopathy
}

\author{
Ingrid Fomison-Nurse and Rajesh Katare
}

Department of Physiology-Heart Otgao, Otago School of Medical Sciences, University of Otago, Dunedin, 9010, New Zealand

*Corresponding author: Rajesh Katare, Department of Physiology-Heart Otago, Otago School of Medical Sciences, University of Otago, Dunedin, 9010, New Zealand, Tel: 006434797292; E-mail: rajesh.katare@otago.ac.nz

Rec date: May 22, 2014, Acc date: June 18, 2014, Pub date: June 26, 2014

Copyright: (c) 2014 Nurse IF et al. This is an open-access article distributed under the terms of the Creative Commons Attribution License, which permits unrestricted use, distribution, and reproduction in any medium, provided the original author and source are credited.

\begin{abstract}
Ischaemic heart disease is an increasingly prevalent disease in the developed world, and accounts for a large degree of both morbidity and mortality in many countries. Diabetes Mellitus is also increasing in prevalence and as ischaemic heart disease is associated with Diabetes Mellitus, diabetic cardiomyopathy is an increasing problem globally. MicroRNAs are short, non-coding RNAs which negatively regulate gene expression through either translational repression or mRNA cleavage. These are found to exist in a stable form in both tissue and blood, and are specific to the tissue of origin. Cardiovascular miRNAs have been found to play roles in cardiac arrhythmogenesis, hypertrophy, and cardiac stem cell differentiation, as well as showing considerable links with diabetic cardiomyopathy. This interaction is important when considering the therapeutic potential of cardiovascular miRNAs, with possible therapeutic value in the treatment of vascular dysfunction, as well as the development of biomarkers for the early diagnosis and therefore treatment of the disease.
\end{abstract}

Keywords: Diabetes; microRNA; Diabetic cardiomyopahy

\section{Introduction}

Ischaemic heart disease is a major cause of death in many developed countries and is becoming more often associated with diabetes mellitus, with cardiovascular disease as one of the primary causes of both morbidity and mortality in patients with diabetes, noted as early as 1970 [1]. As the incidence of diabetes rises, with a projected increase from $14 \%$ in 2010 to $25-28 \%$ in 2050 among United States adults, so does the occurrence of associated heart disease. It is therefore imperative for new and innovative ways to diagnose and treat such complications early on in the disease progression [2].

\section{Diabetic Cardiomyopathy}

Diabetic cardiomyopathy was first described in the literature in a study of post-mortem findings carried out on patients with confirmed diabetic glomerulosclerosis. Of the 27 patients, four were found to have cardiomegaly and congestive heart failure without any obvious cause, including hypertension, coronary artery disease, or valvular diseases. Further analysis of cardiac tissue revealed left ventricular hypertrophy and fibrosis, and with the absence of any other causative factor, this heart disease was characterized as diabetic cardiomyopathy [3].

Left ventricular diastolic dysfunction was described to be an early sign of diabetic cardiomyopathy, with even young insulin-dependent diabetic patients with no apparent systolic dysfunction showing diastolic dysfunction, including reduced diastolic filing, increased atrial filling, and longer times for isovolumetric relaxation and deceleration time [4].

The Multicentre Investigation of the Limitation of Infarct Size (MILIS) study included a comparison of left ventricular function in diabetic and non-diabetic patients with acute infarction. Even after adjusting for the baseline differences between the two groups (with diabetic patients presenting with worsened cardiovascular risk factors), results showed an increase in the incidence of adverse outcomes such as mortality, reduction in ejection fraction, and postoperative complications such as post-infarction angina, further infarcts, and intraventricular conduction delay [5]. This has been further investigated, with one group exploring the effect of diabetes on left ventricular function in patients with aortic stenosis. Peri-operative left ventricular biopsies were obtained along with echocardiography results from patients undergoing aortic valve replacement. Hyperphosphorylation of the stiff $\mathrm{N} 2 \mathrm{~B}$ titin isoform in diabetic patients could explain the increased resting tension of isolated cardiomyocytes. Collagen deposition in the myocardium was also found to be increased, along with glycated end-product deposition in the cardiac vasculature. The combination of all of these factors results in significant left ventricular dysfunction characteristic of diabetic cardiomyopathy [6].

The Framingham study showed that diabetic men of the age bracket 45-74 years had twice the incidence of heart disease than non-diabetic subjects, with diabetic women displaying five times the incidence of heart disease. This risk seemed to be irrespective of other cardiovascular complications and thus supports the characterisation of diabetic cardiomyopathy [7]. Coronary flow is also affected in insulindependent diabetics, as shown by a study by Strauer et al., [8] in which echocardiography was employed to measure cardiac function. Both coronary flow and reserve were significantly reduced whilst coronary resistance was increased. It was also shown that diastolic function was impaired with increased relaxation time and reduced diastolic inflow [8]. Increased deposition of collagen fibres leading to fibrosis causes a stiffening of the ventricular wall, resulting in a reduced ability of the heart muscle to effectively contract or pump $[9,10]$. This translates to a longer relaxation time as previously mentioned coinciding with a reduction in the amount of blood able to flow into the heart. Thus the key feature of diabetic cardiomyopathy seems to involve a loss of function in the left ventricular significantly associated with fibrosis. 


\section{MicroRNAs}

MicroRNAs (miRNAs) are short ( 22 nucleotides), noncoding RNAs that modify protein expression by targeting the 3 ' untranslated region of mRNA, thus regulating specific gene expression at a posttranslational level. An early study into Caenorhabditis elegans development reported that the gene lin-14 controlled the timing of developmental sequences in this species by encoding a nuclear protein present only in early stages of development. Deletion of the 3'untranslated region (present in two types of lin-14 mutation involving gain-of-function) resulted in raised levels of these nuclear proteins in later development stages. This indicated the importance of lin-14 in the temporal switch of development and posed the idea that some factor was responsible for maintaining normal levels of regulatory proteins, potentially through the 3 -untranslated region [11]. The role of the 3'-untranslated region was also reported in 2005, where genes that are involved in basic regulatory cellular processes that were not regulated by miRNA showed short 3'-untranslated regions with a lack of miRNA binding sites [12].

miRNAs were originally identified in the aforementioned C. elegans nematode, in which lin-14 translation was regulated via antisense RNA interactions, but have since been shown to exist in many other organisms extending from single-cell algae to humans [13-17]. Whilst originally thought to have developed along with other multicellular organisms, miRNAs have also been found to exist in the single-cell alga Chlamydomonas reinhardtii, functioning in similar ways to those in multi-cell organisms through RNA silencing and are important in processes ranging from Zebrafish embryonic development to cancer and other disease states [18-20].

The existence of miRNAs in such a wide range of species indicates that miRNA function is not only important but also essential for normal cell development and regulation, thus preserved throughout evolution, with species of higher order development displaying more miRNAs. This importance is also evident in the degree of sequence conservation within and across species. A four-genome analysis of 3' untranslated regions showed regulatory relationships in approximately $30 \%$ of the human gene set, with more than 5300 human genes (one third) as potential conserved miRNA targets [21].

The pathway which forms mature miRNAs has been well studied and consists of various enzymatic steps, as shown in Figure 1. Most miRNAs are transcribed by RNA polymerase II into primary miRNAs (pri-miRNAs), with some, such as the human chromosome cluster of miRNAs, transcribed by polymerase III $[22,23]$. The RNase III Drosha processes the pri-miRNAs in the nucleus, resulting in the formation of pre-miRNAs, which are commonly 70-100 nucleotides long, with a characteristic 'hairpin' structure. RNA interference to deplete Drosha levels in HeLa cells causes a loss of function of Drosha, resulting in a build-up of the pri-miRNA, with reduced levels of pre-miRNAs and mature miRNA in vivo [24]. These pre-miRNAs move into the cytoplasm where the endonuclease Dicer, a member of the RNase III family of nucleases, develops the pre-miRNA into a double stranded miRNA $[25,26]$. This is known as a RNA-Induced Silencing Complex (RISC), which is a negative controller of gene expression, through both translational repression and mRNA cleavage [27]. The specificity of this RNA-induced silencing complex is dependent on the type of miRNA incorporated within it, and thus the gene regulation is dependent on the miRNA (Figure 1).

\section{miRNA Action}

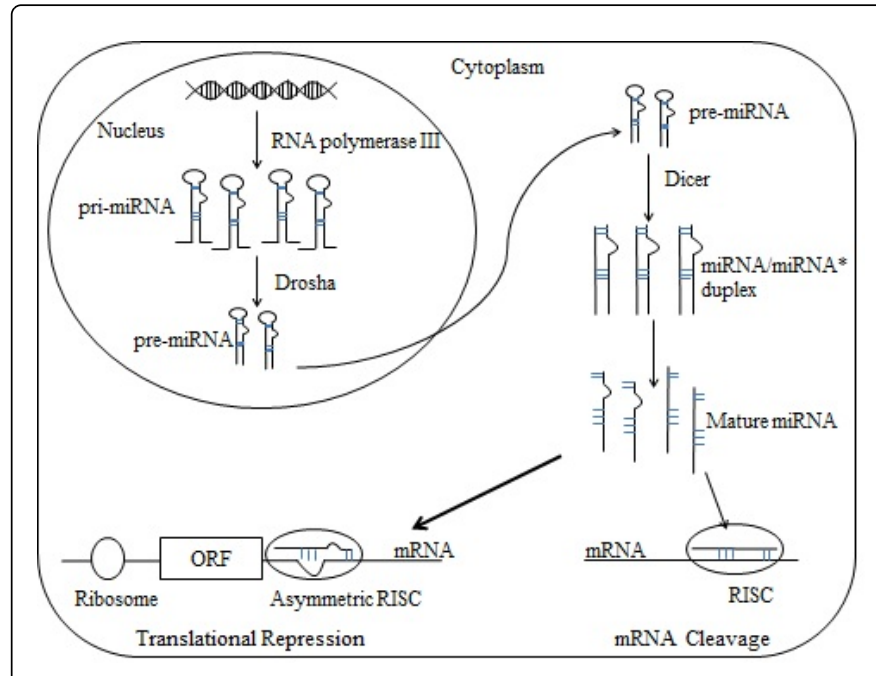

Figure 1: Schematic of the formation of miRNA

\section{miRNA in the Tissue}

Many studies have shown that miRNAs are stable in tissue, plasma, and even urine. One such study reported miRNAs (such as miR-141) deriving from human prostate cancer to be detected in human plasma using RT-PCR assays. This was not significantly altered by extended periods up to 24 hours at room temperature, and these endogenous miRNAs remained stable whilst exogenous miRNAs were degraded by plasma RNases. These levels were similar between plasma and serum [28]. Another group showed that even in total RNA samples damaged by heat incubation the miRNA levels were still detectable with no obvious difference to the intact samples, while the mRNAs decreased as the sample integrity decreased [29]. miRNA profiling in diabetic plasma revealed a reduction in miR-126, a potential factor involved in the impaired peripheral angiogenic signalling characteristic of diabetes, with this also detected using qPCR in both human and mice plasma [30]. Circulating miRNAs are also detectable in other disease states, including gastric cancer, and ST Elevation Myocardial Infarction (STEMI) [31,32]. miRNAs have also been detected in saliva with qPCR performed on saliva samples from oral squamous cell carcinoma patients, with a similar result showing that endogenous miRNA degraded to a lesser extent compared to exogenous miRNA [33].

One study used pigs in a coronary occlusion-reperfusion model to study plasma, urine and tissue miRNA expression. Plasma miR-1, miR-133a, miR-208b, and miR-499-5p were all elevated in the porcine model after coronary occlusion was induced, with significant increases also apparent in the plasma of ST-Elevation Myocardial Infarction (STEMI) patients. These correlated with the glomerular filtration rate, indicating a renal elimination route [32]. MiR-133a levels have also been noted to be elevated in patients with STEMI, with increased miR-133a levels correlating with reduced myocardial salvage, worsened and larger infarcts, and greater reperfusion injury, as reported from a study of 216 patients with STEMI who were undergoing primary angioplasty. While the increase meant that miR-133a levels could be used as a prognostic marker, the levels of miR-133a were not detected any earlier than other current biomarkers 
Page 3 of 6

such as troponin I and troponin $\mathrm{T}$ so could not add independent prognostic information in high-risk populations [34].

As ribonucleases in the plasma would be expected to destroy any miRNA present in the blood, suggestions have been made as to whether miRNAs are protected within vesicles or protein complexes. It was proposed that RNA in the plasma was protected via protein or lipid vesicles, including exosomes, microvesicles, and apoptotic bodies. It was revealed in normal healthy human plasma samples by RT-PCR of the peripheral blood mononuclear cells and plasma microvesicles that miRNAs were co-expressed in both components. Those detected were involved in either blood cell differentiation or immune function, this being the first description of microvesicular compartmentalisation of miRNAs in plasma $[35,36]$. Whilst exosomes and microvesicles would both involve fusing or blebbing of the plasma membrane, release of miRNAs in apoptotic bodies would indicate cell damage severe enough to activate apoptosis, and is thus likely to be relevant in many disease states. However evidence is showing that miRNAs are more likely to be found bound to RNA-binding proteins, forming protein complexes [37].

The clear stability of miRNAs in the plasma begs the question of why the body has developed to allow such stability. Whilst the potential of using miRNAs as biomarkers arises, another hypothesis involves the ability of miRNAs to act in intercellular messengers which could be to modify the destination environment to somehow reflect the environment from which the miRNAs derive.

\section{miRNA Specificity}

Specificity of either the vesicle or the associated protein can indicate where the miRNA originates, and thus most research claims miRNA specificity to certain tissues. Lim et al, 2005, studied the effect of miRNAs on transcription rates in human cells. HeLa cells were transfected with the RNA duplexes of two miRNAs, miR-1 or miR-124. These miRNAs are known for tissue specificity, with miR-1 expressed in heart and skeletal muscle, and miR-124 expressed in the brain $[38,39]$. The mRNA was purified and profiled on microarrays, resulting in down-regulation of 96 annotated genes by miR-1, and 174 genes by miR-124. Genes down-regulated for miR-1 corresponded with the same genes in cardiac and skeletal tissue with endogenously low levels, with the same effect also noted with miR-124. The authors concluded that the addition of particular miRNAs can shift the expression profile to that of the tissue for which a miRNA is specific, indicating that such tissue specificity is linked to the type of genes regulated by the miRNAs [40].

\section{Cardiovascular miRNAs}

As most disease states involve some change in gene expression, the regulation of gene expression by miRNAs is thought to be altered, thus a major focus of miRNA research has been to investigate how specific disease states and miRNAs correlate. Specificity of miRNAs to certain tissues has been well studied, with many cardiovascular miRNAs documented, shown in Table 1.

\begin{tabular}{|l|l|}
\hline MicroRNA & Cardiovascular function \\
\hline miR-1 & Involved in cardiac arrhythmogenesis [41,42]. \\
\hline miR-132 & Regulation of cardiac hypertrophy, as well as roles in cardiomyocyte autophagy [43]. \\
\hline miR-133 & $\begin{array}{l}\text { Involved in cardiac hypertrophy and hyperplasia, with regulation of myocardial matrix remodelling, as well as contributions to } \\
\text { QT interval prolongation in the diabetic heart [44,45]. }\end{array}$ \\
\hline miR-206 & $\begin{array}{l}\text { Involved in cardiac apoptosis during myocardial infarction, as well as a role in cardiac remodelling after a cardiac event } \\
{[46-48] .}\end{array}$ \\
\hline miR-208 & Upregulated in a myocardial infarction [49,50]. \\
\hline miR-499 & Associated with cardiac cell differentiation [51,52]. \\
\hline
\end{tabular}

Table 1: Various cardiac specific microRNAs and their roles in the cardiac system

MiR-1 is considered to be muscle specific and is overexpressed in patients with coronary artery disease [41]. One study used an ischaemia - reperfusion rat model of cardiovascular disease through occlusion of the coronary artery for 30 minutes followed by 24 hours of reperfusion, showing that miR-1 levels in the myocardium were increased while levels of Bcl-2, a key protein involve in apoptosis regulation, were reduced. In in vitro studies based in $\mathrm{H} 9 \mathrm{c} 2$ (a rat ventricular cell line) and HEK293 (human embryonic kidney cell line) it was similarly shown that overexpression of miR-1 resulted in a reduction of Bcl-2 mRNA and protein, which could contribute to increased apoptosis in cardiomyocytes [53].

Induction of cardiac hypertrophy in mice resulted in an increased expression of both miR-212 and miR-132, with evidence pointing toward the subsequent down-regulation of FoxO3 transcription factor, which is known to be both anti-hypertrophic and pro-autophagic [43]. It is clear that apoptosis is a major process affected by miRs in cardiovascular disease.

\section{Cardiovascular miRNAs in Diabetic Cardiomyopathy}

The role of tissue-specific miRNAs in diabetic cardiomyopathy is becoming an increasingly studied field, with many of these miRNAs identified to be involved in the disease. A study published in 2007 measured the expression of 428 miRNAs found in human left ventricular samples from patients with ischaemic cardiomyopathy, dilated cardiomyopathy, or aortic stenosis. It was reported that 43 miRNAs tested showed differential expression, and concluded that different cardiac diseases are associated with different miRNA profiles [54]. Another study used cardiac tissue from the area bordering the infarcted region from patients who were undergoing heart transplant surgery. This tissue also showed differential expression of miRNAs which was similar to the expression profiles found in mice with induced myocardial infarctions [55].

MiRNAs are therefore differentially expressed in human cardiac disease; however research is limited pertaining to the role of miRNAs 
in diabetic heart disease. The interaction of miRNAs and diabetic cardiomyopathy has been investigated further using both in vitro and in vivo techniques. MiR-1 and miR-206 have been reported to play a role in the expression of Hsp60, subsequently contributing to apoptosis mediated by glucose in cardiomyocytes [56]. An increase in miR-1 in diabetic mice has also been correlated with a decrease in Pim-1, a known pro-survival factor which also interacts with HSP-60, with this reduction in Pim-1 associated with further cardiac apoptosis [57]. Diabetic mice with cardiac hypertrophy have been shown to have reduced levels of miR-133a, which could indicate a role of miR-133a in the development of diabetic cardiac hypertrophy [58].

The link between miRs and increased apoptosis demonstrated in cardiac disease is also seen in diabetic cardiomyopathy, with one study reporting in cardiomyocytes exposed to high glucose conditions increased apoptosis, which was linked to the activity miR-1 [59]. Another study investigated the levels of miR-320 in myocardial microvascular endothelial cells from type- 2 diabetic rats, with an increase in miR-320 correlating with a decrease in the expression of insulin-like growth factor 1 which acts to inhibit apoptosis [60]. This evidence, along with the previously mentioned effect of miR-1 on Hsp60 expression in glucose induced cardiac apoptosis, indicates that the increased apoptotic drive seems to be a key component of miRNA involvement in diabetic cardiomyopathy [56].

Mice with streptozotocin-induced diabetes have been shown to develop cardiomyopathy after only 2 months of diabetes duration, with miRNA analysis in cardiac tissue from these mice showing that miR-133a contributes significantly to the characteristic cardiac changes, with down-regulation of miR-133a occurring in diabetic cardiomyopathy. Further in vitro studies in neonatal rat cardiomyocytes using a miR-133a mimic demonstrated the causal relationship of miR-133a in the glucose-induced cardiomyocytes hypertrophy. Results from both in vivo and in vitro models showed that the miR-133a end-targets IGF1R and SGK1 are both up-regulated in diabetic cardiomyopathy which could be a potential way in which miR-133a mediates cardiac hypertrophy in diabetes [58].

Hsp60 is a known component of protection against myocardial injury but is seen to be reduced in the diabetic myocardium. Cardiomyocytes cultured in hyperglycaemic conditions show a significant up-regulation of miR-1 and miR-206, with posttranscriptional modification of Hsp60. Both serum response factor and the MEK $1 / 2$ pathway were also linked to the miR-1 and miR-206 involvement in diabetic cardiomyopathy, although the major role of these particular miRNAs in the associated apoptosis of diabetic cardiomyopathy seems to be through the regulation of Hsp60 [56].

\section{Therapeutic Potential of miRNAs}

The potential of using miRNAs as therapeutic targets in diabetic cardiomyopathy is a relatively new concept in this field. A recent study induced cardiac hypertrophy in mice by TAC and injected antagomirs, oligonucleotides that are complementary to miRNA targets with a mispairing at cleavage sites or base modification to inhibit base cleavage, into these mice intravenously. This successfully knockeddown endogenous miR-132 levels in cardiac tissue, resulting in improved preservation of cardiac function and less dilatation. This could be a potential method to be investigated as a therapeutic intervention to prevent development of hypertrophy and heart failure in diabetic cardiomyopathy [43]. In another study miR-221 was found to be increased in hyperglycaemic conditions in Human Umbilical
Vein Endothelial Cells (HUVECs), mimicking the endothelial cell dysfunction observed in diabetics, a condition which can contribute to diabetic cardiomyopathy. This study went on to postulate that by manipulating the miR-221 and associated c-kit pathway could lead to potential treatment of such vascular dysfunction [61].

A clear advantage of miRNA involvement in diabetic cardiomyopathy is the use of miRNAs as biomarkers for the disease, especially in the earlier stages where diabetic cardiomyopathy often goes unnoticed. Diabetic cardiomyopathy is difficult to diagnose early, as many pathological changes do not manifest as symptoms until later in the disease progression. Diagnostic methods are helpful in general cardiac dysfunction, but are not specific to diabetic cardiomyopathy, with no single tool for this diagnosis readily available $[62,63]$. As the major symptom of diabetic cardiomyopathy is a myocardial infarction, most damage has already occurred, and this damage is irreversible.

Several miRNAs, including miR-454, miR-500, miR-1246, and miR-142-3p/5p, have been identified in patients with diastolic dysfunction in plasma samples, using standard miRNA profiling techniques, with different profiles of up or down regulation in the disease state seen among the different miRNAs [64].

miRNAs are a new and exciting field in the context of disease states, with much research investigating the roles they play in diseases including cancer and cardiomyopathies. By modifying protein expression through negative control of gene expression, miRNAs have the ability to alter significant cellular processes. This is part of normal cellular activity but has been shown to become deregulated in various disease states, becoming both markers for disease, as well as causing further damage. The potential for these relatively tissue-specific regulatory molecules to be used as biomarkers and even therapeutic targets for diseases such as diabetic cardiomyopathy is a widely promising research focus, and remains an open question to be answered. There is a great need for further research in this area, with the role of miRNAs in human diabetic cardiomyopathy an emerging and exciting topic of research.

\section{References}

1. Garcia MJ, McNamara PM, Gordon T, Kannel WB (1974) Morbidity and mortality in diabetics in the Framingham population. Sixteen year follow-up study. Diabetes 23: 105-111.

2. Boyle JP, Honeycutt AA, Narayan KM, Hoerger TJ, Geiss LS, et al. (2001) Projection of diabetes burden through 2050: impact of changing demography and disease prevalence in the U.S. Diabetes Care 24: 1936-1940.

3. Rubler S, Dlugash J, Yuceoglu YZ, Kumral T, Branwood AW, et al. (1972) New type of cardiomyopathy associated with diabetic glomerulosclerosis. Am J Cardiol 30: 595-602.

4. Schannwell CM1, Schneppenheim M, Perings S, Plehn G, Strauer BE (2002) Left ventricular diastolic dysfunction as an early manifestation of diabetic cardiomyopathy. Cardiology 98: 33-39.

5. Stone PH, Muller JE, Hartwell T, York BJ, Rutherford JD, et al. (1989) The effect of diabetes mellitus on prognosis and serial left ventricular function after acute myocardial infarction: contribution of both coronary disease and diastolic left ventricular dysfunction to the adverse prognosis. Journal of the American College of Cardiology 14:49-57.

6. Falcão-Pires I, Hamdani N, Borbély A, Gavina C, Schalkwijk CG et al. (2011) Diabetes Mellitus Worsens Diastolic Left Ventricular Dysfunction in Aortic Stenosis Through Altered Myocardial Structure and Cardiomyocyte Stiffness. Clinical Perspective. Circulation 124:1151-1159.

7. Kannel WB, Hjortland M, Castelli WP (1974) Role of diabetes in congestive heart failure: the Framingham study. Am J Cardiol 34: 29-34. 
8. Strauer BE, Motz W, Vogt M, Schwartzkopff B (1997) Evidence for reduced coronary flow reserve in patients with insulin-dependent diabetes. A possible cause for diabetic heart disease in man. Exp Clin Endocrinol Diabetes 105: 15-20.

9. Sutton MG, Sharpe N (2000) Left ventricular remodeling after myocardial infarction: pathophysiology and therapy. Circulation 101: 2981-2988.

10. Shirani J, Pick R, Roberts WC, Maron BJ (2000) Morphology and significance of the left ventricular collagen network in young patients with hypertrophic cardiomyopathy and sudden cardiac death. Journal of the American College of Cardiology 35:36-44.

11. Wightman B, Bürglin T, Gatto J, Arasu P, Ruvkun G (1991) Negative regulatory sequences in the lin-14 3'-untranslated region are necessary to generate a temporal switch during Caenorhabditis elegans development. Genes \& development 5:1813-1824.

12. Stark A, Brennecke J, Bushati N, Russell RB, Cohen SM (2005) Animal MicroRNAs confer robustness to gene expression and have a significant impact on 3'UTR evolution. Cell 123: 1133-1146.

13. Lee RC, Feinbaum RL, Ambros V (1993) The C. elegans heterochronic gene lin-4 encodes small RNAs with antisense complementarity to lin-14. Cell 75: 843-854.

14. Zhao Y, Samal E, Srivastava D (2005) Serum response factor regulates a muscle-specific microRNA that targets Hand2 during cardiogenesis. Nature 436: 214-220.

15. Tang G-Q, Maxwell ES (2008) Xenopus microRNA genes are predominantly located within introns and are differentially expressed in adult frog tissues via post-transcriptional regulation. Genome research 18: 104-112.

16. Jeyaseelan K, Lim KY, Armugam A (2008) MicroRNA expression in the blood and brain of rats subjected to transient focal ischemia by middle cerebral artery occlusion. Stroke 39: 959-966.

17. Chen JF, Mandel EM, Thomson JM, Wu Q, Callis TE, et al. (2006) The role of microRNA-1 and microRNA-133 in skeletal muscle proliferation and differentiation. Nat Genet 38: 228-233.

18. Molnár A, Schwach F, Studholme DJ, Thuenemann EC, Baulcombe DC (2007) miRNAs control gene expression in the single-cell alga Chlamydomonas reinhardtii. Nature 447: 1126-1129.

19. Wienholds E, Koudijs MJ, van Eeden FJ, Cuppen E, Plasterk RH (2003) The microRNA-producing enzyme Dicer1 is essential for zebrafish development. Nat Genet 35: 217-218.

20. Wienholds E, Kloosterman WP, Miska E, Alvarez-Saavedra E, Berezikov E, et al. (2005) MicroRNA expression in zebrafish embryonic development. Science 309: 310-311.

21. Lewis BP, Burge CB, Bartel DP (2005) Conserved seed pairing, often flanked by adenosines, indicates that thousands of human genes are microRNA targets. Cell 120: 15-20.

22. Lee Y, Kim M, Han J, Yeom KH, Lee S, et al. (2004) MicroRNA genes are transcribed by RNA polymerase II. EMBO J 23: 4051-4060.

23. Borchert GM, Lanier W, Davidson BL (2006) RNA polymerase III transcribes human microRNAs. Nat Struct Mol Biol 13: 1097-1101.

24. Lee Y, Ahn C, Han J, Choi H, Kim J, et al. (2003) The nuclear RNase III Drosha initiates microRNA processing. Nature 425: 415-419.

25. Bernstein E, Caudy AA, Hammond SM, Hannon GJ (2001) Role for a bidentate ribonuclease in the initiation step of RNA interference. Nature 409: 363-366.

26. Grishok A, Pasquinelli AE, Conte D, Li N, Parrish S, et al. (2001) Genes and mechanisms related to RNA interference regulate expression of the small temporal RNAs that control C. elegans developmental timing. Cell 106: 23-34.

27. Liu Q, Rand TA, Kalidas S, Du F, Kim HE, et al. (2003) R2D2, a bridge between the initiation and effector steps of the Drosophila RNAi pathway. Science 301: 1921-1925.

28. Mitchell PS, Parkin RK, Kroh EM, Fritz BR, Wyman SK, et al. (2008) Circulating microRNAs as stable blood-based markers for cancer detection. Proc Natl Acad Sci U S A 105: 10513-10518.
29. Jung M, Schaefer A, Steiner I, Kempkensteffen C, Stephan C, et al. (2010) Robust microRNA stability in degraded RNA preparations from human tissue and cell samples. Clin Chem 56: 998-1006.

30. Zampetaki A, Kiechl S, Drozdov I, Willeit P, Mayr U, et al. (2010) Plasma microRNA profiling reveals loss of endothelial miR-126 and other microRNAs in type 2 diabetes. Circ Res 107: 810-817.

31. Tsujiura M, Ichikawa D, Komatsu S, Shiozaki A, Takeshita H, et al. (2010) Circulating microRNAs in plasma of patients with gastric cancers. Br J Cancer 102: 1174-1179.

32. Gidlöf O, Andersson P, Van Der Pals J, Götberg M, Erlinge D (2011) Cardiospecific microRNA plasma levels correlate with troponin and cardiac function in patients with ST elevation myocardial infarction, are selectively dependent on renal elimination, and can be detected in urine samples. Cardiology 118: 217-226.

33. Park NJ, Zhou H, Elashoff D, Henson BS, Kastratovic DA, et al. (2009) Salivary microRNA: discovery, characterization, and clinical utility for oral cancer detection. Clin Cancer Res 15: 5473-5477.

34. Eitel I, Adams V, Dieterich P, Fuernau G, de Waha S, et al. (2012) Relation of circulating MicroRNA-133a concentrations with myocardial damage and clinical prognosis in ST-elevation myocardial infarction. Am Heart J 164: 706-714.

35. El-Hefnawy T, Raja S, Kelly L, Bigbee WL, Kirkwood JM, et al. (2004) Characterization of amplifiable, circulating RNA in plasma and its potential as a tool for cancer diagnostics. Clin Chem 50: 564-573.

36. Hunter MP, Ismail N, Zhang X, Aguda BD, Lee EJ, et al. (2008) Detection of microRNA expression in human peripheral blood microvesicles. PLoS One 3: e3694.

37. Hafner M, Landthaler M, Burger L, Khorshid M, Hausser J, et al. (2010) Transcriptome-wide identification of RNA-binding protein and microRNA target sites by PAR-CLIP. Cell 141: 129-141.

38. Lagos-Quintana M, Rauhut R, Yalcin A, Meyer J, Lendeckel W, et al. (2002) Identification of tissue-specific microRNAs from mouse. Curr Biol 12: 735-739.

39. Sempere LF, Freemantle S, Pitha-Rowe I, Moss E, Dmitrovsky E et al. (2004) Expression profiling of mammalian microRNAs uncovers a subset of brain-expressed microRNAs with possible roles in murine and human neuronal differentiation. Genome Biol 5: R13.

40. Lim LP, Lau NC, Garrett-Engele P, Grimson A, Schelter JM, et al. (2005) Microarray analysis shows that some microRNAs downregulate large numbers of target mRNAs. Nature 433: 769-773.

41. Yang B, Lin H, Xiao J, Lu Y, Luo X, et al. (2007) The muscle-specific microRNA miR-1 regulates cardiac arrhythmogenic potential by targeting GJA1 and KCNJ2. Nat Med 13: 486-491.

42. Terentyev D, Belevych AE, Terentyeva R, Martin MM, Malana GE, et al. (2009) miR-1 overexpression enhances $\mathrm{Ca} 2+$ release and promotes cardiac arrhythmogenesis by targeting PP2A regulatory subunit B56a and causing CaMKII-dependent hyperphosphorylation of RyR2. Circulation research 104: 514-521.

43. Ucar A, Gupta SK, Fiedler J, Erikci E, Kardasinski M, et al. (2012) The miRNA-212/132 family regulates both cardiac hypertrophy and cardiomyocyte autophagy. Nat Commun 3: 1078.

44. Duisters RF, Tijsen AJ, Schroen B, Leenders JJ, Lentink V, et al. (2009) miR-133 and miR-30 regulate connective tissue growth factor: implications for a role of microRNAs in myocardial matrix remodeling. Circ Res 104: 170-178, 6p following 178.

45. Xiao J, Luo X, Lin H, Zhang Y, Lu Y, et al. (2007) MicroRNA miR-133 represses HERG $\mathrm{K}+$ channel expression contributing to QT prolongation in diabetic hearts. J Biol Chem 282: 12363-12367.

46. Shan ZX, Lin QX, Fu YH, Deng CY, Zhou ZL, et al. (2009) Upregulated expression of miR-1/miR-206 in a rat model of myocardial infarction. Biochem Biophys Res Commun 381: 597-601.

47. Kim HK, Lee YS, Sivaprasad U, Malhotra A, Dutta A (2006) Musclespecific microRNA miR-206 promotes muscle differentiation. J Cell Biol 174: 677-687. 
Citation: Nurse I F, Katare R (2014) Emerging Roles of Micrornas in Diabetic Cardiomyopathy. J Diabetes Metab 5: 394 . doi:

Page 6 of 6

48. Limana F, Esposito G, D'Arcangelo D, Di Carlo A, Romani S, et al. (2011) HMGB1 attenuates cardiac remodelling in the failing heart via enhanced cardiac regeneration and miR-206-mediated inhibition of TIMP-3. PLoS One 6: e19845.

49. Ji X, Takahashi R, Hiura Y, Hirokawa G, Fukushima Y, et al. (2009) Plasma miR-208 as a biomarker of myocardial injury. Clin Chem 55 1944-1949.

50. Bostjancic E, Zidar N, Stajer D, Glavac D (2010) MicroRNAs miR-1, miR-133a, miR-133b and miR-208 are dysregulated in human myocardial infarction. Cardiology 115: 163-169.

51. Zhang LL, Liu JJ, Liu F, Liu WH, Wang YS, et al. (2012) MiR-499 induces cardiac differentiation of rat mesenchymal stem cells through wnt $/ \hat{\mathrm{I}}^{2}$ catenin signaling pathway. Biochem Biophys Res Commun 420: 875-881.

52. Wilson KD, Hu S, Venkatasubrahmanyam S, Fu JD, Sun N, et al. (2010) Dynamic microrna expression programs during cardiac differentiation of human embryonic stem cells role for mir-499. Circulation: Cardiovascular Genetics 3: 426-435.

53. Tang Y, Zheng J, Sun Y, Wu Z, Liu Z, et al. (2009) MicroRNA-1 regulates cardiomyocyte apoptosis by targeting Bcl-2. Int Heart J 50: 377-387.

54. Ikeda S, Kong SW, Lu J, Bisping E, Zhang H, et al. (2007) Altered microRNA expression in human heart disease. Physiol Genomics 31: 367-373.

55. van Rooij E, Sutherland LB, Thatcher JE, DiMaio JM, Naseem RH, et al (2008) Dysregulation of microRNAs after myocardial infarction reveals a role of miR-29 in cardiac fibrosis. Proc Natl Acad Sci U S A 105: 13027-13032.

56. Shan ZX, Lin QX, Deng CY, Zhu JN, Mai LP, et al. (2010) miR-1/ miR-206 regulate Hsp60 expression contributing to glucose-mediated apoptosis in cardiomyocytes. FEBS Lett 584: 3592-3600.
57. Katare R, Caporali A, Zentilin L, Avolio E, Sala-Newby G, et al. (2011) Intravenous gene therapy with PIM-1 via a cardiotropic viral vector halts the progression of diabetic cardiomyopathy through promotion of prosurvival signaling. Circ Res 108: 1238-1251.

58. Feng B, Chen S, George B, Feng Q, Chakrabarti S (2010) miR133a regulates cardiomyocyte hypertrophy in diabetes. Diabetes Metab Res Rev 26: 40-49.

59. Yu XY, Song YH, Geng YJ, Lin QX, Shan ZX, et al. (2008) Glucose induces apoptosis of cardiomyocytes via microRNA-1 and IGF-1. Biochem Biophys Res Commun 376: 548-552.

60. Wang XH, Qian RZ, Zhang W, Chen SF, Jin HM, et al. (2009) MicroRNA-320 expression in myocardial microvascular endothelial cells and its relationship with insulin-like growth factor- 1 in type 2 diabetic rats. Clin Exp Pharmacol Physiol 36: 181-188.

61. Li Y, Song YH, Li F, Yang T, Lu YW, et al. (2009) MicroRNA-221 regulates high glucose-induced endothelial dysfunction. Biochem Biophys Res Commun 381: 81-83.

62. Hayat SA, Patel B, Khattar RS, Malik RA (2004) Diabetic cardiomyopathy: mechanisms, diagnosis and treatment. Clin Sci (Lond) 107: 539-557.

63. Maya L, Villarreal FJ (2010) Diagnostic approaches for diabetic cardiomyopathy and myocardial fibrosis. J Mol Cell Cardiol 48: 524-529.

64. Nair N, Kumar S, Gongora E, Gupta S (2013) Circulating miRNA as novel markers for diastolic dysfunction. Mol Cell Biochem 376: 33-40. 